\title{
Sexualidad en la mujer histerectomizada: modelo predictivo al sexto mes posterior a la cirugía
}

\author{
María Teresa U rrutia $S^{1 a}$, Alejandra Araya $\mathbf{G}^{1 b}$, Soledad \\ Rivera ${ }^{1 c}$, Paola Viviani2d, Luis Villarroel2d. \\ A predictive model for the quality of \\ sexual life in hysterectomized women
}

Background: The effects of hysterectomy on sexuality has been extensively studied. Aim: To establish a model to predict the quality of sexual life in hysterectomized women, six months after surgery. Material and Methods: Analytical, longitudinal and prospective study of 90 hysterectomized women aged $45 \pm 7$ years. Two structured interviews at the time of surgery and six months later were carried out to determine the characteristics of sexuality and communication within the couple. Results: In the two interviews, communication and the quality of sexual life were described as "good" in 72 and $77 \%$ of women, respectively (NS). The variables that had a $40 \%$ influence on the quality of sexual life sixth months after surgery, were oophorectomy status, the presence of orgasm, the characteristics of communication and the basal sexuality with the couple. Conclusions: The sexuality of the hysterectomized women will depend, on a great extent, of pre-surgical variables. Therefore, it is important to consider these variables for the education of hysterectomized women (Rev Méd Chile 2007; 135: 317-25).

(Key words: Hysterectomy; Sexual behavior; Sexuality)

Recibido el 27 de enero, 2006. Aceptado el 22 de agosto, 2006.

Proyecto financiado por la Dirección de Investigación de la Escuela de Enfermería de la Pontificia Universidad Católica de Chile.

${ }^{1}$ Escuela de Enfermería y ${ }^{2}$ Departamento de Salud Pública, Facultad de Medicina, Pontificia Universidad Católica de Chile.

aEnfermera Matrona, Magíster en Nutrición.

bEnfermera Matrona, Licenciada en Enfermería.

'Enfermera, Doctora en Enfermería, Magíster en Salud Pública.

dEstadístico.

$\mathrm{E}^{\mathrm{n}}$ n los países desarrollados, la histerectomía (HT) es la segunda cirugía más frecuente después de la cesárea ${ }^{1,2}$. En Estados Unidos de

Correspondencia a: EU María Teresa Urrutia. Vicuña Mackenna 4860. Escuela de Enfermería, Campus San Joaquín, Macul. Santiago. Fax: 3547025-3547910. E mail: murrutis@uc.cl
Norteamérica, 40,5\% de las mujeres a los 64 años se encontrará histerectomizada ${ }^{3}$; en Chile, se estima que $20 \%$ de las mujeres en algún momento de la vida será sometida a esta cirugía ${ }^{4}$. El mayor porcentaje de HT es por causa benigna y la principal patología es la miomatosis uterina ${ }^{5,6}$.

La sexualidad de las mujeres histerectomizadas es dinámica y la evolución de los cambios posterior a la cirugía ${ }^{5}$, no excedería los 6 meses 
posoperatorios $^{7,8}$. Se ha descrito, una mantención de la frecuencia de relaciones sexuales ${ }^{9}$, un aumento de la libido y una disminución de la frecuencia de dolor y de problemas sexuales ${ }^{10-12}$ posterior a esta cirugía. Sin embargo, se ha reportado $20 \%$ a $25 \%$ de las mujeres con un deterioro en su vida sexual ${ }^{13}$ y en la percepción de su feminidad, atractivo y deseo sexual ${ }^{14}$.

En una investigación nacional, señalan que el tercer mes posterior a la HT sería una etapa de transición entre la sexualidad preoperatoria y la definitiva ${ }^{15}$. En este estudio las variables frecuencia de orgasmo, percepción de deseo sexual, grado de excitación y satisfacción sexual no presentan diferencias significativas entre el estado basal y el tercer mes. Sin embargo, la frecuencia de relaciones sexuales y la presencia de orgasmo muestran diferencias, siendo menores las frecuencias de ambas variables al tercer mes poshisterectomía. Se realizó un seguimiento al sexto mes ${ }^{16}$, en donde las variables anteriormente señaladas no presentaron diferencias significativas; la satisfacción sexual mejoró significativamente. Por lo anterior, las autoras señalan, que la sexualidad de la mujer no se ve afectada en las mujeres histerectomizadas ${ }^{16}$.

Existen múltiples publicaciones en torno a la vida sexual de la mujer histerectomizada, sin embargo, el mayor porcentaje de ellas mide sólo aspectos disfuncionales. La valoración sexual debe considerar una perspectiva más amplia, trascendiendo la medición netamente funcional», $\mathrm{y}$, por tanto, apuntando al concepto de calidad de vida sexual.

El objetivo de esta investigación es establecer un modelo que permita predecir la calidad de la vida sexual de la mujer histerectomizada al sexto mes posterior a la cirugía.

\section{MATERIAL Y MÉTODO}

Estudio analítico, longitudinal, prospectivo, realizado en un grupo de mujeres histerectomizadas del Servicio de Ginecología del Hospital Dr. Sótero del Río, perteneciente al Servicio de Salud Metropolitano Sur-Oriente de Santiago. Ingresaron al estudio todas las mujeres histerectomizadas durante el periodo comprendido entre diciembre del año 2002 y abril de 2003. A modo de referencia, durante el año 2002, se realizaron 420 histerectomías. Los criterios de inclusión fueron: patología ginecológica benigna, vida sexual activa en los últimos tres meses, pareja estable en los últimos tres meses, ausencia de patología psiquiátrica diagnosticada o en tratamiento, histerectomía de causa ginecológica, y deseo de participar. No se consideró excluir a las mujeres por otra causa, dada la necesidad de conocer el fenómeno nunca antes estudiado en nuestro país. De un universo de 177 mujeres histerectomizadas, 104 mujeres cumplieron con los criterios de inclusión. Las variables estudiadas comprenden las características descriptivas de las mujeres, antecedentes de la cirugía y de la patología causante de la histerectomía, calidad de comunicación de pareja y calidad de vida sexual; las dos últimas variables fueron medidas al tercer día posoperatorio y al sexto mes después de la histerectomía; el lapso de tiempo de 6 meses se decidió basado en publicaciones que señalan que la recuperación sexual de las mujeres no excedería este periodo de tiempo ${ }^{7,8}$. La entrevista basal fue realizada en el hospital, en una oficina privada; la del sexto mes, en el domicilio de la usuaria. En ambos casos la persona encargada fue la misma, con el objetivo de no incomodar a la usuaria. La entrevista fue realizada por enfermeras-matronas e internas de enfermeríaobstetricia. Para la recolección de los datos de calidad de comunicación de pareja y calidad de vida sexual, se realizó una adaptación del instrumento «ercepción de Calidad de Comunicación de pareja y de Calidad de Vida Sexual $\gg^{17}$, previa autorización de la autora, cuyo Alpha de Cronbach es de 0,95 para la escala de calidad de vida sexual y 0,89 para la escala de calidad de comunicación de pareja. El instrumento original fue aplicado en una muestra de mujeres chilenas provenientes del mismo servicio de salud. La adaptación para este estudio consideró, fundamentalmente, seleccionar aquellas preguntas más representativas de las diferentes dimensiones medidas en el instrumento original, junto con excluir aquellas preguntas relacionadas a sistema de valores sexuales y antecedentes traumáticos sexuales, variables no estudiadas en esta investigación. La adaptación, si bien puede ser otra limitación de este estudio, fue necesaria, dado que no existen instrumentos nacionales que midan calidad de vida sexual en mujeres histerectomizadas. El instrumento adaptado arrojó un Alpha de Cronbach de 0,86 para la escala de calidad de 
comunicación de pareja y de 0,86 para la de calidad de vida sexual. La escala calidad de comunicación de pareja abarca las siguientes dimensiones: expresión de deseo, acogida, facilitación y percepción general de la comunicación de pareja actual; consta de 9 preguntas tipo Likert, con un puntaje máximo de 45 puntos y un mínimo de 9 puntos, a mayor puntaje es peor la comunicación de pareja. La escala de calidad de vida sexual abarca las dimensiones: excitación, penetración, orgasmo, resolución, percepción de deseo, evitación, aceptación, angustia y evaluación general de la vida sexual actual, con un total de 15 preguntas tipo Likert; el puntaje máximo es 71 puntos y el mínimo de 15, a mayor puntaje es peor la calidad de vida sexual. Ambas escalas permiten clasificar el puntaje en tres rangos: buena, regular y mala. Se solicitó consentimiento informado a cada una de las mujeres previo a la entrevista, en donde se explicita la posibilidad de que pueda sentirse incómoda hablando de su vida

\section{Tabla 1. C aracterísticas descriptivas de la población} en estudio $(n=90)$

\begin{tabular}{|lcc|}
\hline & Promedio \pm DS & $\%$ \\
\hline Edad & $45,5 \pm 6,7$ & - \\
Escolaridad & $8,9 \pm 3,3$ & - \\
Años de relación de pareja & $20,6 \pm 10,22$ & - \\
Estado civil casada & - & 76 \\
\hline
\end{tabular}

Tabla 2. D istribución porcentual de las mujeres según causa de la histerectomía ( $n=90) *$

\begin{tabular}{|lrr|}
\hline $\begin{array}{l}\text { Causa de la } \\
\text { histerectomía }\end{array}$ & $\begin{array}{r}\text { Frecuencia } \\
(\mathrm{n})\end{array}$ & $\begin{array}{r}\text { Porcentaje } \\
(\%)\end{array}$ \\
\hline Miomatosis uterina & 60 & 52,2 \\
Prolapso genital & 17 & 14,8 \\
Tumor anexial & 12 & 10,4 \\
Incontinencia urinaria & & \\
$\quad$ de esfuerzo & 11 & 9,6 \\
Endometriosis & 7 & 6,1 \\
Algia pélvica & 5 & 4,3 \\
Absceso tubo-ovárico & 3 & 2,6 \\
Total & 115 & 100 \\
\hline
\end{tabular}

* La frecuencia total es mayor a 90 ya que algunas mujeres presentan más de una causa. sexual y de pareja; sin embargo, se asegura la confidencialidad de la información entregada y que se velará a lo largo de todo el proceso para no causar incomodidad, dada la naturaleza del estudio. La presente investigación fue aprobada por el comité de ética de la Escuela de Enfermería de la Pontificia Universidad Católica de Chile.

Estadística. Los datos fueron analizados a través del programa SPSS 12.0; se utilizaron medidas de tendencia central y dispersión, análisis de frecuencia, T Student para muestra pareadas, test de Kappa, regresión lineal univariada y regresión lineal múltiple. Se consideró significativo un valor $\mathrm{p}<0,05$.

\section{RESULTADOS}

De las 104 mujeres ingresadas, hubo una pérdida de $13,5 \%$ durante el seguimiento, por lo que el análisis de datos fue realizado en 90 mujeres. Las causas de pérdida de las 14 mujeres fueron: 9 cambiaron de pareja, 1 se le diagnosticó cáncer de colon y 4 no lograron ser ubicadas.

Las características descriptivas del grupo se presentan en la Tabla 1. Del total de la muestra, 10 mujeres $(11,2 \%)$ se encontraban menopaúsicas previo a la HT. En relación a las características de la cirugía, se realizó HT total en 89 casos y sólo 1 HT subtotal. En 75,6\%, la vía de abordaje fue abdominal y el restante vía vaginal. La ooforectomía fue realizada en $58,9 \%$, siendo bilateral en $81,1 \%$ de este grupo. Al sexto mes poscirugía ninguna de las mujeres era usuaria de terapia de reemplazo hormonal (TRH). La causa principal de HT fue la miomatosis uterina (Tabla 2).

Algunas características de la sexualidad, que no fueron medidas a través del instrumento de calidad de vida sexual de las mujeres de este estudio, tanto al tercer mes como al sexto posterior a la cirugía, han sido previamente publica$\operatorname{das}^{15,16}$, como son la frecuencia de relaciones sexuales, presencia y frecuencia de orgasmo, prevalencia de incontinencia y de dispareunia.

En relación a la calidad de comunicación de pareja, los resultados señalan una muy leve variación porcentual, al compararla en los dos periodos de tiempo (Tabla 3). Sin embargo, la diferencia de promedios no es significativa (valor 
Tabla 3. D istribución porcentual de las mujeres en estudio según calidad de comunicación de pareja

\begin{tabular}{|c|c|c|c|c|c|}
\hline \multirow{2}{*}{$\begin{array}{l}\text { Comunicación de } \\
\text { Pareja }\end{array}$} & \multirow{2}{*}{ Puntaje } & \multicolumn{2}{|c|}{ Entrevista basal } & \multicolumn{2}{|c|}{ Entrevista 6 meses } \\
\hline & & $\mathrm{n}$ & $(\%)$ & $\mathrm{n}$ & $(\%)$ \\
\hline Buena & $9-20$ & 65 & 72,2 & 62 & 68,9 \\
\hline Regular & $21-33$ & 21 & 23,3 & 26 & 28,9 \\
\hline Mala & $34-45$ & 4 & 4,4 & 2 & 2,2 \\
\hline Total & & 90 & 100 & 90 & 100 \\
\hline
\end{tabular}

Valor $\mathrm{p}=0,750$.

$\mathrm{p}=0,750)$. Al comparar las respuestas de la entrevista basal con las del sexto mes, todas las dimensiones del instrumento se mantuvieron sin diferencias significativas, salvo una pregunta de la dimensión facilitación. La diferencia de esta pregunta hace referencia a un menor número de peleas percibidas por las mujeres al sexto mes, en comparación a la entrevista basal (Tabla 4).

Tabla 4. D imensiones y preguntas de la escala calidad de comunicación de pareja (escala Likert)

\begin{tabular}{|c|c|c|c|}
\hline Dimensión & Pregunta & Test Kappa & Valor $\mathrm{p}$ \\
\hline \multirow[t]{2}{*}{$\begin{array}{l}\text { Expresión de } \\
\text { deseo }\end{array}$} & $\begin{array}{l}\text { Cuando su compañero está en } \\
\text { problemas cél se acerca a } \\
\text { pedirle apoyo? }\end{array}$ & 0,184 & 0,012 \\
\hline & $\begin{array}{l}\text { Cuando usted está en } \\
\text { problemas ¿usted se acerca a } \\
\text { pedirle apoyo? }\end{array}$ & 0,247 & 0,000 \\
\hline \multirow[t]{2}{*}{ Acogida } & $\begin{array}{l}\text { Cuando conversan ¿siente } \\
\text { usted que él la escucha? }\end{array}$ & 0,410 & 0,000 \\
\hline & $\begin{array}{l}\text { ¿Cuando conversan usted } \\
\text { lo escucha? }\end{array}$ & 0,211 & 0,007 \\
\hline \multirow[t]{2}{*}{ Facilitación } & $\begin{array}{l}\text { ¿Siente usted que es difícil } \\
\text { conversar con su compañero? }\end{array}$ & 0,298 & 0,000 \\
\hline & $\begin{array}{l}\text { ¿Sus conversaciones terminan } \\
\text { en peleas? }\end{array}$ & 0,126 & $0,080^{*}$ \\
\hline \multirow[t]{4}{*}{$\begin{array}{l}\text { Percepción } \\
\text { general }\end{array}$} & $\begin{array}{l}\text { Actualmente custed encuentra } \\
\text { su matrimonio (relación de } \\
\text { pareja)? }\end{array}$ & 0,100 & 0,033 \\
\hline & $\begin{array}{l}\text { ¿Su vida matrimonial } \\
\text { (relación de pareja) actual es } \\
\text { para usted agradable? }\end{array}$ & 0,298 & 0,000 \\
\hline & $\begin{array}{l}\text { Actualmente ¿El matrimonio } \\
\text { (relación de pareja) resulta } \\
\text { satisfactorio para usted? }\end{array}$ & 0,303 & 0,000 \\
\hline & $\begin{array}{l}\text { ¿Siente usted que su } \\
\text { matrimonio (relación de } \\
\text { pareja) es estable? }\end{array}$ & 0,348 & 0,001 \\
\hline
\end{tabular}

* Concordancia no significativa. 
En relación a la calidad de vida sexual, el análisis se realizó en 86 mujeres, ya que cuatro mujeres no habían iniciado actividad sexual al sexto mes; las causas eran: dos de ellas por temor al dolor, una por problemas de comunicación de pareja y la cuarta por la incontinencia urinaria, secundaria a la histerectomía.

La Tabla 5 presenta los puntajes y clasificación de la calidad de vida sexual percibida por la mujer en ambos periodos de tiempo. Al analizar el puntaje total de la escala, no se encontraron diferencias significativas entre la medición basal y al sexto mes (valor $\mathrm{p}=0,106$ ). Al analizar cada una de las dimensiones con sus respectivas preguntas, no hubo diferencias en la mayoría de las respuestas de vida sexual basal y al sexto mes. Las respuestas que variaron son de la dimensión excitación, angustia y evaluación general de la vida sexual, todas ellas apuntan a una mejoría de los parámetros evaluados (Tabla 6).

Para la selección de las variables ingresadas al modelo multivariado, se realizó un análisis univariado, considerando como variable dependiente la calidad de vida sexual al sexto mes. Las variables que resultaron significativamente relacionadas con la vida sexual al sexto mes fueron: calidad de vida sexual basal, comunicación de pareja basal, frecuencia de orgasmo, años de escolaridad y edad de la mujer.

Para el análisis multivariado se ingresaron las variables: edad de la mujer, años de escolaridad, presencia de ooforectomía, presencia de dismenorrea, patología asociada, tipo de HT, frecuencia de relaciones, presencia y frecuencia de dispareunia, presencia y frecuencia de orgasmo, calidad de comunicación de pareja basal, calidad de vida sexual basal. La elección de las variables a ingresar al análisis univariado como al multivariado, se realizó en base a variables descritas en la literatura. Del total de variables ingresadas al análisis multivariado, las aceptadas en el modelo fueron: calidad de comunicación de pareja basal, calidad de la sexualidad basal, presencia de ooforectomía y presencia de orgasmo basal (Tabla 7). El modelo establecido explica 39,5\% de la variable calidad de vida sexual al sexto mes post HT.

\section{DISCUSIÓN}

La presente investigación es el primer estudio chileno que propone un modelo predictivo de sexualidad en la mujer histerectomizada al sexto mes después de la cirugía y forma parte de un estudio, con publicaciones previas, en torno a la evolución de la sexualidad de la mujer, al tercer ${ }^{15}$ y sexto mes posterior a la $\mathrm{HT}^{16}$. La lectura de estos resultados deben considerar la limitación que los instrumentos originales que fueron adaptados para este estudio, Percepción de Calidad de Comunicación de pareja y de Calidad de Vida Sexual» (Rivera MS. Vida sexual de mujeres de estrato social medio bajo y bajo y su relación con la comunicación y conflictos de pareja, sistema de valores sexuales y antecedentes traumáticos sexuales. Tesis de Magister en Salud Pública, Mención epidemiología. Universidad de Chile. Santiago, Chile. Disponible en Biblioteca de la Escuela de Salud Pública de la Universidad de Chile. 1986) (Trabajo no publicado).

Es importante señalar que, el porcentaje de mujeres que no han iniciado vida sexual al sexto mes podría ser interpretado como efecto negativo

\section{Tabla 5. D istribución porcentual de las mujeres en estudio según calidad de vida sexual basal y al sexto mes posterior a la cirugía}

\begin{tabular}{|lcrrrr|}
\hline & & \multicolumn{2}{c}{ Entrevista basal } & \multicolumn{2}{c|}{ Entrevista 6 meses } \\
Validad de & & & & \\
Vida Sexual & Puntaje & $\mathrm{n}$ & $(\%)$ & $\mathrm{n}$ & $(\%)$ \\
\hline Buena & $15-33$ & 66 & 76,7 & 67 & 77,9 \\
Regular & $34-52$ & 16 & 18,6 & 19 & 22,1 \\
Mala & $53-71$ & 4 & 4,7 & 0 & 0,0 \\
Total & & 90 & 100 & 86 & 100 \\
\hline
\end{tabular}

Valor $\mathrm{p}=0,106$. 
Tabla 6. D imensiones y preguntas de calidad de vida sexual (escala Likert)

\begin{tabular}{|c|c|c|c|}
\hline Dimensión & Pregunta & $\begin{array}{r}\text { Test } \\
\text { Kappa }\end{array}$ & Valor $\mathrm{p}$ \\
\hline \multirow[t]{4}{*}{ Excitación } & $\begin{array}{l}\text { La mayoría de sus relaciones sexuales } \\
\text { le resultan }\end{array}$ & 0,081 & $0,263 *$ \\
\hline & $\begin{array}{l}\text { Antes de la relación sexual, su pareja la } \\
\text { acaricia }\end{array}$ & 0,306 & 0,000 \\
\hline & Acepta usted que su esposo la acaricie & 0,073 & $0,383^{*}$ \\
\hline & $\begin{array}{l}\text { La excitación que usted alcanza en la mayoría } \\
\text { de las relaciones sexuales es }\end{array}$ & 0,297 & 0,000 \\
\hline Penetración & $\begin{array}{l}\text { Cuando su esposo la penetra, custed está } \\
\text { preparada? }\end{array}$ & 0,342 & 0,000 \\
\hline Orgasmo & $\begin{array}{l}\text { Siente que él se interesa en que usted logre } \\
\text { placer durante la relación sexual }\end{array}$ & 0,466 & 0,000 \\
\hline Resolución & $\begin{array}{l}\text { Después del orgasmo ustedes permanecen } \\
\text { juntos }\end{array}$ & 0,216 & 0,010 \\
\hline $\begin{array}{l}\text { Percepción de } \\
\text { deseo }\end{array}$ & Diría usted que su apetito o deseo sexual es & 0,213 & 0,006 \\
\hline Evitación & $\begin{array}{l}\text { Actualmente usted le saca la vuelta a las } \\
\text { relaciones sexuales }\end{array}$ & 0,286 & 0,000 \\
\hline \multirow[t]{2}{*}{ Aceptación } & $\begin{array}{l}\text { Usted acepta las relaciones sexuales sólo por } \\
\text { complacer a su pareja }\end{array}$ & 0,331 & 0,000 \\
\hline & $\begin{array}{l}\text { Que su compañero le solicite relaciones } \\
\text { sexuales a usted le resulta }\end{array}$ & 0,264 & 0,001 \\
\hline Angustia & $\begin{array}{l}\text { ¿Siente usted angustia intensa antes de iniciar } \\
\text { la relación sexual? }\end{array}$ & 0,070 & $0,333^{*}$ \\
\hline Evaluación & Usted considera que su vida sexual actual es & 0,130 & $0,112^{*}$ \\
\hline $\begin{array}{l}\text { general } \\
\text { de la vida } \\
\text { sexual actual }\end{array}$ & $\begin{array}{l}\text { Si usted no tuviera relaciones sexuales ¿Se } \\
\text { sentiría? }\end{array}$ & 0,144 & $0,064^{*}$ \\
\hline
\end{tabular}

* Concordancia no significativa.

Tabla 7. Modelo predictivo regresión Stepwise: Calidad de vida sexual al sexto mes posterior a la histerectomía

\begin{tabular}{|lr|}
\hline Variable & R Parcial \\
\hline Calidad de vida sexual inicial & 0,189 \\
Ooforectomía & 0,085 \\
Presencia de orgasmo & 0,070 \\
Comunicación de pareja inicial & 0,051 \\
\hline
\end{tabular}

*El modelo explica $39,5 \%$ de la variable.

de la sexualidad, sin embargo, al analizar las causas, nos encontramos con que corresponden a problemas que pudieron haber sido tratados antes de la cirugía o durante el seguimiento, a través de una educación integral centrada en las necesidades de las mujeres y sus parejas. Menos de la mitad de las mujeres discuten aspectos de sexualidad con los profesionales ${ }^{6}$, y aquellas que reciben información la consideran insuficiente ${ }^{17}$. Respecto de aquella mujer que refiere mala comunicación de pareja, ésta es una variable relacionada a la sexualidad de la mujer posterior a la $\mathrm{HT}^{18}$.

La medición de la variable comunicación de pareja resulta de relevancia en esta investigación, ya que según algunos autores, es el mejor predictor de la vida sexual y, por lo tanto, la razón más frecuente de su deterioro es la mala comunicación de pareja ${ }^{18}$. Esta variable no presentó cambios en el grupo de mujeres estudiadas, lo que podría por tanto consti- 
tuirse en un factor favorecedor de una buena sexualidad en este grupo de mujeres. Cabe señalar que en este estudio sólo las mujeres fueron consultadas en torno a la percepción de comunicación de pareja. Resultará interesante presentar futuras investigaciones que consideren la comunicación de pareja desde la perspectiva masculina.

Con respecto a la vida sexual de las mujeres, los resultados encontrados son similares a lo reportado por publicaciones internacionales,3,3,6,19.

En relación a las variables significativas en el análisis de regresión simple y que no emergieron en el análisis de la regresión múltiple, los resultados son coincidentes con publicaciones extranjeras. La literatura señala que la frecuencia de orgasmo es un buen predictor de la sexualidad posterior a la cirugía 20,21 . Existen autores que plantean que un nivel educacional mayor se correlaciona a un aumento de la satisfacción sexual ${ }^{5}$; también ha sido relacionado al tipo de tratamiento elegido por las usuarias: las mujeres con menor nivel educacional prefieren la HT, mientras que aquellas con mayor nivel, prefieren tratamiento farmacológico ${ }^{22}$. Estas variables son importantes de tener en consideración por los profesionales de la salud, ya que pueden influir en la habilidad de la mujer para incorporar el significado de la cirugía en su vida ${ }^{5} \mathrm{y}$, por lo tanto, en la forma de enfrentar este proceso.

La relación entre histerectomía y función sexual considera factores físicos, psicológicos y sociales ${ }^{17}$, reafirmando el concepto que la respuesta sexual femenina es compleja y multidimensional ${ }^{5,17,23}$. Los hallazgos del modelo propuesto señalan que el predictor de calidad de vida sexual al sexto mes poshisterectomía más importante, es la calidad de vida sexual basal. Esto concuerda con lo establecido por otros estudios en donde se señala como el principal factor a considerar ${ }^{20}$. Este antecedente debe incentivar a los profesionales de la salud a indagar en este factor en el periodo preoperatorio, para de esta forma lograr una valoración completa de la mujer.

Con respecto a la ooforectomía, la literatura es contradictoria, ya que por algunos autores es considerada como un factor que influye significativamente en la obtención de resultados insatisfactorios posteriores a la HT, producto del cese hormonal ${ }^{5,9,24}$, sin embargo, otras revisiones realizadas muestran que estos resultados dependen de cada mujer 22,25 . Cabe señalar que la presencia de ooforectomía como factor predictor de la sexualidad, debe ser analizado considerando que ninguna de las mujeres en el grupo de estudio era usuaria de TRH al sexto mes; por lo anterior se hace necesario contar con estudios que permitan dilucidar el real efecto de la ooforectomía, y despejar el de la ausencia de TRH, ya que está descrito que dicha terapia disminuye el riesgo de disfunciones sexuales ${ }^{26}$. Cabe señalar que la ooforectomía fue una variable que en el análisis univariado no resultó significativa, sin embargo emergió en el modelo multivariado; este resultado puede verse explicado por la interrelación que existe entre las diferentes variables, lo que provoca que aquellas que no son importantes en el análisis univariado, lo sean al considerar el peso relativo en presencia de otras variables.

Según el modelo establecido, la sexualidad de las mujeres histerectomizadas dependerá, en gran medida, de variables prequirúrgicas como son la buena calidad de vida sexual, la buena calidad de comunicación de pareja y la presencia de orgasmo, junto con la presencia de ooforectomía, siendo esta última el único predictor negativo de la calidad de vida sexual en nuestro modelo. Los profesionales deben tener en consideración estas variables predictivas al momento de entregar educación a las usuarias ${ }^{21,22}$. La educación de las mujeres histerectomizadas es parte de su cuidado integral ${ }^{17}$. Las mujeres manifiestan ansiedad por el futuro de su vida sexual ${ }^{8}$ y por su relación de pareja después de la cirugía ${ }^{26}$, además de destacar la falta de información entregada sobre su patología, las complicaciones y cambios derivados de la cirugía y la necesidad de tener un apoyo, no sólo por parte de su pareja, sino también por parte de los profesionales de la salud ${ }^{17,27}$. Es esencial conocer las necesidades educativas de las mujeres $^{15,16}$, para proveer un cuidado apropiado ${ }^{7,28} \mathrm{y}$ destinado a preservar, de la mejor manera, la vida sexual.

Si bien el modelo propuesto explica 39,5\% de la variable sexualidad al sexto mes, dicho valor señala que existe $60,5 \%$ que es explicada por variables que no fueron consideradas en el presente estudio; este resultado, por lo tanto, reafirma el concepto de que la sexualidad es una variable compleja de abordar, no incluyendo tan sólo aspectos físicos, sino también emocionales y aquellos propios de la 
identidad de mujer ${ }^{28-30}$. Este resultado hay que leerlo con la precaución necesaria, debido a la limitación expuesta sobre ambos instrumentos originales adaptados para este estudio. Es, por lo tanto, necesario actualizar y publicar este estudio, ya que constituye una fuente para el estudio más integral de la sexualidad, al ligarlo a comunicación de pareja y otras variables como conflictos de pareja, antecedentes traumáticos sexuales y sistema de valores sexuales. También debemos ahondar en otros aspectos no estudiados y que pudieran tener relación con la sexualidad de la mujer histerectomizada. Creemos importante incorporar investigaciones cualitativas en esta área, que permitan conocer las experiencias vividas de las mujeres, desde su percepción y de esta manera, poder complementar el cuerpo de conocimientos ya construido, que nos permita ahondar en las necesidades de las mujeres

\section{REFERENCIAS}

1. Brown J, Sawaya G, Thom D, Grady D. Hysterectomy and urinary incontinence: a systematic review. Lancet 2000; 356: 535-9.

2. Thakar R, Ayers S, Ciarkson P, Stanton S, Manyonda I. Outcomes after total versus subtotal abdominal hysterectomy. N Engl J Med 2002; 347: 1318-25.

3. SCHAFFER J, Word A. Hysterectomy-still a useful operation. N Engl J Med 2002; 347: 1360-2.

4. Ahumada A, Aluaga P, Bolínar N. Enfoque integral de la paciente histerectomizada. Revista Hospital Clínico Universidad de Chile 2001; 12: 229-34.

5. FARRELL S, KIESER K. Sexuality after hysterectomy. Obstet Gynecol 2000; 95: 1045-51.

6. Elustrom M, Astrom M, Moler A, Olsson J, Hahli M. A randomized trial comparing changes in psychological well-being and sexuality after laparoscopic and abdominal hysterectomy. Acta Obstet Gynecol Scand 2003; 82: 871-5.

7. Rannestad T, EIKeland O, Heliand H, Qvarnstrom U. The quality of life in women suffering from gynecological disorders is improved by means of hysterectomy. Absolute and relative differences between pre- and postoperative measures. Acta Obstet Gynecol Scand 2001; 80: 46-51.

8. Roovers J, Van der Bom J, Van der Vaart C, Heintz A. Hysterectomy and sexual wellbeing: prospecti- histerectomizadas ${ }^{31}$, identificando nuevas variables que no han sido consideradas en este estudio y de esta forma orientar en forma más focalizada programas de educación destinados a ellas.

Además, es importante señalar, que si bien es el primer estudio chileno en mujeres histerectomizadas, es necesario realizar nuevas investigaciones donde se considere un seguimiento a largo plazo, para poder analizar variables como el efecto del hipoestrogenismo secundario a la ooforectomía, anteriormente estudiado en nuestro país ${ }^{26}$. Los resultados obtenidos pueden verse influenciados por el alto porcentaje de mujeres ooforectomizadas bilaterales sin TRH, lo que hace necesario plantear futuros estudios en torno al seguimiento y comparación de mujeres histerectomizadas con y sin ooforectomía lateral y a su vez con y sin TRH.

ve observational study of vaginal hysterectomy, subtotal abdominal hysterectomy, and total abdominal hysterectomy. BMJ 2003; 327: 774-8.

9. Rhodes J, KJerulfF K, Langenberg P, Guzinski G. Hysterectomy and sexual functioning. JAMA 1999; 282: 1934-41.

10. Lambden M, Bewamy G, Ogburn-Russell L, Preece C, Moore S, Pepin T ET al. Women's sense of wellbeing before and after hysterectomy. JOGNN 1997; 26: 540-8.

11. Thakar R, Manyonda I, Stanton S, Clarkson P, RoBinson G. Bladder, bowel and sexual function after hysterectomy for benign conditions. $\mathrm{Br} \mathrm{J}$ Obstet Gynaecol 1997; 104: 983-7.

12. Roovers J, Van der Vaart C, Van der Bom J, Van Leeuwen J, Scholten P, Heintz A. A randomized controlled trial comparing abdominal and vaginal prolapse surgery: effects on urogenital function. BJOG 2004; 111: 50-6.

13. LeMACK G, Zimmern P. Sexual function after vaginal surgery for stress incontinence: results of a mailed questionnaire. Urology 2000; 56: 223-7.

14. Heiman J, Meston C. Evaluating sexual dysfunction in women. Clin Obstet Gynecol 1997; 40: 616-29.

15. Urrutia M, Araya A. Sexualidad en mujeres histerectomizadas al tercer mes posterior a la cirugía. Rev Chil Obstet Ginecol 2005; 70: 160-5.

16. Urrutia M, Araya A, Viliarroel L, Viñales D. Características y evolución de la sexualidad en 
mujeres histerectomizadas. Rev Chil Obstet Ginecol 2004; 69: 301-6.

17. Wade J, Pletsch P, Morgan S, Menting S. Hysterectomy: what do women need and want to know? JOGNN 2000; 29: 33-42.

18. Helstrom L, Sorbom D, Backstrom T. Influence of partener relationship and sexuality after subtotal hysteretomy. Obstet Gynecol Surv 1996; 51: 224-5.

19. Zobbe V, Gimbel $H$, Andersen B, Filtenborg $T$, JaKobSEN K, SORENSEN H ET aL. Sexuality after total vs subtotal hysterectomy. Acta Obstet Gynecol Scand 2004; 83: 191-6.

20. Helstrom L, Backstrom T, Sorbom D, Lundberg P. Sacral nervous function, hormonal levels and sexuality in premenopausal women before and after hysterectomy. Acta Obstet Gynecol Scand 1994; 73: 570-4.

21. Helstrom L, Lundberg PO, Sorbom D, Backstrom T. Sexuality after hysterectomy: a factor analysis of women's sexual lives before and after subtotal hysterectomy. Obstet Gynecol 1993; 81: 357-62.

22. KIM K, LeE K. Symptom experience in women after hysterectomy. JOGNN 2001; 30: 472-80.

23. KHASTGIR G, Studd J. Patients' outlook, experience, and satisfaction with hysterectomy, bilateral oophorectomy, and subsequent continuation of hormone replacement therapy. Am J Obstet Gynecol 2000; 183: 1427-33.

24. KJeRulfF KH, Langenberg PW, Rhodes JC, Harvey LA, GuZinski GM, Stowey PD. Effectiveness of hysterectomy. Obstet Gynecol 2000; 95: 319-26.

25. Shoupe D. Hysterectomy or an alternative? Hosp Pract (Off Ed) 2000; 35: 55-62.

26. Blumel J, araya H, Riquelme R, Castro G, Sánchez F, Gramegna G. Prevalencia de trastornos de la sexualidad en mujeres climatéricas. Influencia de la menopausia y de la terapia de reemplazo hormonal. Rev Méd Chile 2002; 130: 1131-8.

27. Chapple A. Hysterectomy: British National Health Service and private patients have very different experiences. J Adv Nurs 1995; 22: 900-6.

28. BHAVNAN V, CiarKe A. Women awaiting hysterectomy: a qualitative study of issues involved in decisions about oophorectomy. BJOG 2003; 110: 168-74.

29. Augustus C. Beliefs and perceptions of African American women who have had hysterectomy. J Transcult Nurs 2002; 13: 296-302.

30. BERNHARD L. Sexuality and sexual health care for women. Clin Obstet Gynecol 2002; 45: 1089-98.

31. Katz A. Sexuality after hysterectomy: a review of the literature and discussion of nurses' role. J Adv Nurs 2003; 42: 297-303.

Agradecimientos

A cada una de las mujeres que participaron en este estudio, por la generosidad de compartir sus experiencias. 